\title{
Impact of frailty on outcomes after discharge in older surgical patients: a prospective cohort study
}

\author{
Yibo Li BMSc, Jenelle L. Pederson MSc, Thomas A. Churchill PhD, Adrian S. Wagg MBBS, Jayna M. Holroyd-Leduc MD, \\ Kannayiram Alagiakrishnan MD, Raj S. Padwal MD MSc, Rachel G. Khadaroo MD PhD
}

Cite as: CMAJ 2018 February 20;190:E184-90. doi: 10.1503/cmaj.161403

See related article at www.cmaj.ca/lookup/doi/10.1503/cmaj.170902

\section{ABSTRACT \\ BACKGROUND: Frailty is a state of vulner- ability to diverse stressors. We assessed the impact of frailty on outcomes after discharge in older surgical patients.}

METHODS: We prospectively followed patients 65 years of age or older who underwent emergency abdominal surgery at either of 2 tertiary care centres and who needed assistance with fewer than 3 activities of daily living. Preadmission frailty was defined according to the Canadian Study of Health and Aging Clinical Frailty Scale as "well" (score 1 or 2), "vulnerable" (score 3 or 4) or "frail" (score 5 or 6). We assessed composite end points of 30-day and 6-month all-cause readmission or death by multivariable logistic regression.
RESULTS: Of 308 patients (median age 75 [range 65-94] yr, median Clinical Frailty Score 3 [range 1-6]), 168 (54.5\%) were classified as vulnerable and $68(22.1 \%)$ as frail. Ten (4.2\%) of those classified as vulnerable or frail received a geriatric consultation. At 30 days after discharge, the proportions of patients who were readmitted or had died were greater among vulnerable patients $(n=27[16.1 \%]$; adjusted odds ratio [OR] 4.60, 95\% confidence interval $[\mathrm{Cl}] 1.29-16.45)$ and frail patients ( $n=12$ [17.6\%]; adjusted OR 4.51, 95\% Cl 1.13-17.94) than among patients who were well ( $n=3[4.2 \%])$. By 6 months, the degree of frailty independently and dose-dependently predicted readmission or death: 56 (33.3\%) of the vulnerable patients (adjusted OR 2.15, $95 \% \mathrm{Cl} 1.01-4.55)$ and 37 (54.4\%) of the frail patients (adjusted OR 3.27, 95\% Cl 1.32-8.12) were readmitted or had died, compared with $11(15.3 \%)$ of the patients who were well.

INTERPRETATION: Vulnerability and frailty were prevalent in older patients undergoing surgery and unlikely to trigger specialized geriatric assessment, yet remained independently associated with greater risk of readmission for as long as 6 months after discharge. Therefore, the degree of frailty has important prognostic value for readmission. Trial registration for primary study: ClinicalTrials. gov, no. NCT02233153

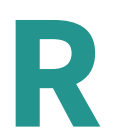

eadmissions are expensive, ${ }^{1}$ have been considered an important quality indicator for surgical care ${ }^{2}$ and are highest after abdominal procedures. ${ }^{1}$ Older patients are increasingly being admitted with acute surgical conditions ${ }^{3}$ and have a higher risk of readmission. ${ }^{4}$

Frailty is more prevalent among, although it is not limited to, older patients. ${ }^{5}$ It is widely accepted that frailty is a multifactorial state that is marked by vulnerability to internal and external stress $^{6}$ and that may change over time. ${ }^{6,7}$ Frailty is a known risk factor for complications, ${ }^{8-11}$ prolonged hospital stay ${ }^{8,12}$ and adverse discharge disposition. ${ }^{8,11}$ Furthermore, degree of frailty shows a dose-response relation to mortality in both surgical ${ }^{13}$ and critically ill ${ }^{5}$ patients. However, the impact of frailty on readmission after surgery in older patients has rarely been assessed.

Measuring frailty in hospital using a rapid tool may be espe- cially valuable for surgeons who treat older patients whose risk of poor outcomes is not captured by their age alone. ${ }^{6,14}$ Perhaps the greatest opportunity lies in increasing recognition of patients who are at high risk without noticeable disability, which could enable early intervention. ${ }^{6}$ The Canadian Study of Health and Aging Clinical Frailty Scale ${ }^{15}$ is a previously validated 9-point global subjective assessment of comorbidity and function that does not require specialized training or entail extensive assessment; ${ }^{16}$ it has surpassed more complex frailty assessments in predicting readmission and mortality. ${ }^{17,18}$

Very few studies have examined the risk associated with frailty for subsequent health care utilization in any surgical group. ${ }^{18}$ Therefore, we assessed the impact of preadmission frailty on 30-day and 6-month readmission or death in older patients undergoing emergency abdominal surgery. 


\section{Methods}

\section{Population and data collection}

We report here a substudy of patients who were prospectively enrolled during the pre-implementation phase of the Elderfriendly Approaches to the Surgical Environment (EASE) study (clinicaltrials.gov identifier NCT02233153). ${ }^{19}$ We included patients aged 65 years or older who survived emergency abdominal surgery at 2 tertiary care hospitals in Canada (University of Alberta Hospital, Edmonton, and Foothills Medical Centre, Calgary) between January 2014 and September 2015. Patients who required assistance with 3 or more activities of daily living, underwent palliative or trauma surgery, or were transferred from another ward or hospital were excluded. Baseline and demographic characteristics were collected by trained research assistants through chart review and patient interviews in the hospital or at follow-up. We calculated the Charlson Comorbidity Index for each patient; this index has previously been validated for acutely ill older patients. ${ }^{20}$

\section{Main independent variable}

We assessed frailty using the revised Canadian Study of Health and Aging Clinical Frailty Scale. ${ }^{15}$ Trained research assistants determined frailty status in the 2 weeks preceding index admission by interviewing patients (or their surrogates) shortly after admission or by reviewing medical charts. Degree of frailty was defined as very fit to well (termed "well," for brevity; score 1 or 2), managing well to vulnerable (termed "vulnerable;" score 3 or 4) or mildly to moderately frail (termed "frail;" score 5 or 6). "Very fit" refers to people who are very active and energetic; "well" indicates those who are occasionally active; "managing well" indicates those who are physically inactive beyond routine walking; "vulnerable" indicates those with comorbidity and limited activity, but without disability; "mild frailty" indicates dependence in 1 or more instrumental activities of daily living (e.g., food preparation or housework); and "moderate frailty" indicates dependence in 1 or 2 basic activities of daily living (e.g., dressing, bathing). ${ }^{16}$ Patients with severe or very severe frailty, indicating complete dependence in 3 or more activities of daily living, and terminally ill patients (score $\geq 7$ ) were ineligible for the EASE study.

\section{Outcomes}

The primary outcome was composite all-cause readmission or death at 30 days and 6 months after discharge, which accounts for the competing risk of death. Readmission and mortality data were collected from a province-wide electronic medical records database. Readmission was defined as acute care admission after the initial surgery, excluding transfers for rehabilitation or convalescence.

\section{Statistical analysis}

We determined descriptive statistics, calculating proportions, means and medians. We identified some potential confounders a priori on the basis of existing literature and clinical importance (age, sex and type of surgery). Additional potential confounders were identified in univariable analyses that compared baseline covariables with readmission or death and with frailty, with a predefined cut-off of $p<0.2$. We used Fisher exact tests for categorical variables and $t$ tests or one-way analysis of variance for continuous variables (see Table 1 for the variables included). Trends for covariables and degree of frailty are reported.

We then developed multivariable logistic regression models using a standard approach ${ }^{21}$ to calculate the odds of readmission or death. Patients who were readmitted or who died were considered to have had an event and were included in the numerator to account for the competing risk of death. Age, sex and type of surgery were forced into all models. Identified potential confounders were then sequentially entered into the model and retained if they met statistical criteria for confounding (potential confounder $p<0.1$ or $>10 \%$ change in the frailty $\beta$-coefficient upon inclusion). We chose the most parsimonious models, allowing as many as 6 variables at 30 days and 7 variables at 6 months. Model fit was judged using the Hosmer-Lemeshow goodness-of-fit test, and accuracy was judged using the $C$ statistic. We conducted sensitivity analyses to assess the relation of interest per 1-point increase in the Clinical Frailty Scale and the additional prognostic value of increasing age and American Society of Anesthesiologists classification (https://www.asahq.org/resources/clinical-information/ asa-physical-status-classification-system). We also conducted a post hoc sensitivity analysis excluding patients who underwent cancer surgery. We considered the Clinical Frailty Scale as a dichotomized variable, applying a well-balanced cut-off score of 4 (rather than 5, as used previously ${ }^{5,22,23}$ ), because our cohort excluded patients with scores of 7 or higher. Finally, we disaggregated composite outcomes to identify whether readmission or death was driving the relation.

Statistical significance was defined on the basis of 2-tailed $p<0.05$. All analyses were conducted with Stata 14 software (StataCorp LP).

\section{Ethics approval}

The University of Alberta Research Ethics Board (Pro00047180) and the University of Calgary Research Ethics Board (REB140729) approved the study procedures.

\section{Results}

Of 3506 patients screened, most were excluded because of age younger than 65, conservative management or transfer (Figure 1). We enrolled 322 patients and retrieved readmission or mortality data for all patients. Fourteen of the patients died before discharge and were thus excluded from the analyses (Figure 1).

\section{Cohort characteristics}

The median age was 75 (range 65-94) years, and 140 (45.4\%) of the 308 patients were women. The median score on the Clinical Frailty Scale was 3 (range 1-6): 15 (4.9\%) of the patients were "very fit" (score = 1), 57 (18.5\%) were "well" (score = 2), 108 $(35.1 \%)$ were "managing well" (score = 3), 60 (19.5\%) were "vulnerable" (score =4), 39 (12.7\%) had "mild frailty" (score = 5), and $29(9.4 \%)$ had "moderate frailty" (score $=6)$. Before the index 
Table 1: Baseline characteristics of older patients discharged from hospital after emergency

abdominal surgery

Preadmission level of frailty*; no. (\%) of patients $\dagger$

\begin{tabular}{|c|c|c|c|}
\hline $\begin{array}{c}\text { Well } \\
n=72\end{array}$ & $\begin{array}{c}\text { Vulnerable } \\
\qquad n=168\end{array}$ & $\begin{array}{c}\text { Frail } \\
n=68\end{array}$ & $p$ value $\ddagger$ \\
\hline $73.9 \pm 7.0$ & $75.2 \pm 6.7$ & $79.9 \pm 9.2$ & $<0.001$ \\
\hline $30(41.7)$ & 75 (44.6) & 35 (51.5) & 0.2 \\
\hline $57(79.2)$ & $122(72.6)$ & $52(76.5)$ & 0.7 \\
\hline $52(72.2)$ & $122(72.6)$ & $37(54.4)$ & 0.03 \\
\hline $0(0-1)$ & $1(0-2)$ & $2(1-3)$ & $<0.001$ \\
\hline $26 \pm 4.5$ & $27.6 \pm 6.4$ & $26.9 \pm 6.5$ & 0.4 \\
\hline $1.3 \pm 0.5$ & $1.5 \pm 0.6$ & $2.0 \pm 0.7$ & $<0.001$ \\
\hline $57(79.2)$ & $115(68.5)$ & $37(54.4)$ & 0.003 \\
\hline $60(83.3)$ & $138(82.1)$ & $50(73.5)$ & 0.1 \\
\hline $64(88.9)$ & $133(79.2)$ & $57(83.8)$ & 0.3 \\
\hline \multirow[t]{2}{*}{$61(84.7)$} & $113(67.3)$ & $36(52.9)$ & $<0.001$ \\
\hline & & & 0.01 \\
\hline $7(9.7)$ & $25(14.9)$ & $11(16.2)$ & \\
\hline $19(26.4)$ & $44(26.2)$ & $24(35.3)$ & \\
\hline $10(13.9)$ & $23(13.7)$ & $9(13.2)$ & \\
\hline $10(13.9)$ & $9(5.4)$ & $4(5.9)$ & \\
\hline $23(31.9)$ & $52(31.0)$ & $11(16.2)$ & \\
\hline $3(4.2)$ & $15(8.9)$ & $9(13.2)$ & \\
\hline $2(2-3)$ & $3(2-3)$ & $3(3-4)$ & $<0.001$ \\
\hline $3(4.2)$ & $16(9.5)$ & $9(13.2)$ & 0.06 \\
\hline $68(94.4)$ & $153(91.1)$ & $48(70.6)$ & $<0.001$ \\
\hline $0(0-1)$ & $0(0-1)$ & $1(0-2)$ & $<0.001$ \\
\hline $1(1.4)$ & $3(1.8)$ & $7(10.3)$ & 0.005 \\
\hline $14(19.4)$ & $33(19.6)$ & $21(30.9)$ & 0.1 \\
\hline $41(56.9)$ & $111(66.1)$ & $51(75.0)$ & 0.02 \\
\hline \multirow[t]{2}{*}{$7(4-11)$} & $9(6-12)$ & $13(7.5-27.5)$ & $<0.001$ \\
\hline & & & $<0.001$ \\
\hline 63 (87.5) & $121(72.0)$ & $24(35.3)$ & \\
\hline $7(9.7)$ & $33(19.6)$ & $17(25.0)$ & \\
\hline $2(2.8)$ & $11(6.5)$ & $24(35.3)$ & \\
\hline $0(0)$ & $2(1.2)$ & $3(4.4)$ & \\
\hline
\end{tabular}

Note: $\mathrm{ASA}=$ American Society of Anesthesiologists, $\mathrm{BMI}=$ body mass index, IQR = interquartile range, $\mathrm{SD}=$ standard deviation *Level of frailty based on Canadian Study of Health and Aging Clinical Frailty Scale. ${ }^{15}$

†Except where indicated otherwise.

$\ddagger$ Based on $\chi^{2}$ tests of trend using linear regression for continuous variables, score test of trend of odds for binary variables and Wilcoxon-type test of trend for categorical variables.

$\S$ Variables identified as potential confounders in the univariable analyses. The following additional variables, for which data are not shown, were also included in the univariable analyses: current smoking status, vital signs on admission, blood glucose level and white blood cell count on admission, and major surgical complication. 
admission, $228(74.0 \%)$ of the patients had been living at home independently, $62(20.1 \%)$ had been living at home with assistance, 16 (5.2\%) had been residing in a nursing home, and 2 $(0.6 \%)$ had other living arrangements. The mean Charlson Comorbidity Index was 1.1 (standard deviation 1.2), and nearly all (292 [94.8\%]) of the patients underwent a single surgery during their admission; 14 patients $(4.5 \%)$ required a second surgery, and $2(0.6 \%)$ required more than 2 procedures. Patients underwent the following types of surgery: 109 (35.4\%) had cholecystectomy or appendectomy, $87(28.2 \%)$ had small intestinal surgery, $43(14.0 \%)$ had colon surgery, 42 (13.6\%) had hernia repair, and $27(8.8 \%)$ had some other type of abdominal surgery.

\section{Prevalence and degree of frailty}

Seventy-two (23.4\%) of the patients were classified as well (score 1 or 2$), 168(54.5 \%)$ as vulnerable (score 3 or 4$)$, and $68(22.1 \%)$ as frail (score 5 or 6 ) (Table 1). The patients classified as vulnerable or frail were older, had a higher number of comorbidities and medications, and more often presented with abnormal serum hemoglobin and creatinine levels than patients classified as well (Table 1).

At surgery, patients classified as frail were more likely than those classified as well or vulnerable to undergo a laparoscopic (closed) approach for cholecystectomy or appendectomy; if they had intestinal surgery, they were more likely to receive an ostomy (Table 1). Patients classified as vulnerable or frail scored a median of 3 on the American Society of Anesthesiologists classification (interquartile range [IQR] 2-3 and 3-4, respectively), compared with 2 (IQR 2-3) for patients classified as well (Table 1). Although the American Society of Anesthesiologists classification was sta-

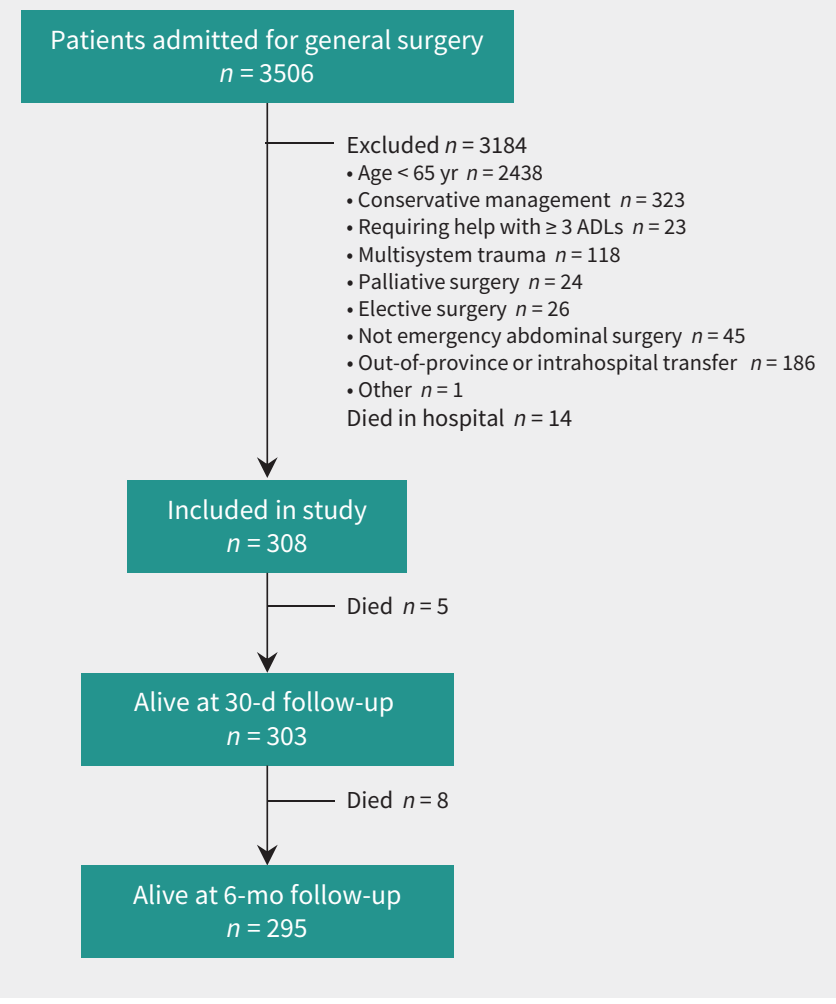

Figure 1: Selection of study patients. ADLs = activities of daily living. tistically different for different levels of frailty, the median and mode were the same for patients classified as vulnerable or frail (data not shown). Patients classified as frail were more likely to require postoperative intensive care or close observation and to stay in hospital longer and were less likely to be discharged home independently (Table 1). Although patients classified as vulnerable or frail more often underwent a geriatric assessment than patients classified as well, overall only $4.2 \%(10 / 236)$ of the former group were assessed by a geriatrician.

\section{Thirty-day readmission or death}

Within 30 days after discharge, $42(13.6 \%)$ of the patients were readmitted or had died. These outcomes occurred in $27(16.1 \%)$ of those classified as vulnerable and $12(17.6 \%)$ of those classified as frail, but only $3(4.2 \%)$ of those classified as well ( $p=0.02$; Figure 2, Table 2). After adjustment for age, sex, type of surgery, abnormal hemoglobin level and postoperative use of total parenteral nutrition, vulnerable status remained associated with 30-day readmission or death (adjusted odds ratio [OR] 4.60, 95\% confidence interval [Cl] 1.29-16.45), as did frail status (adjusted OR 4.51, 95\% Cl 1.13-17.94; C statistic 0.75, 95\% Cl 0.67-0.83) (Table 2).

\section{Six-month readmission or death}

By 6 months after discharge, 104 (33.8\%) of the patients were readmitted or had died. These outcomes occurred in 56 (33.3\%) of those classified as vulnerable and 37 (54.4\%) of those classified as frail, but only $11(15.3 \%)$ of those classified as well ( $p<$ 0.001; Figure 2, Table 2). After adjustment for age, sex, type of surgery, abnormal hemoglobin level, Charlson Comorbidity Index and intraoperative ostomy creation, degree of frailty predicted 6-month readmission or death in a dose-dependent manner (for patients classified as vulnerable, adjusted OR 2.15,

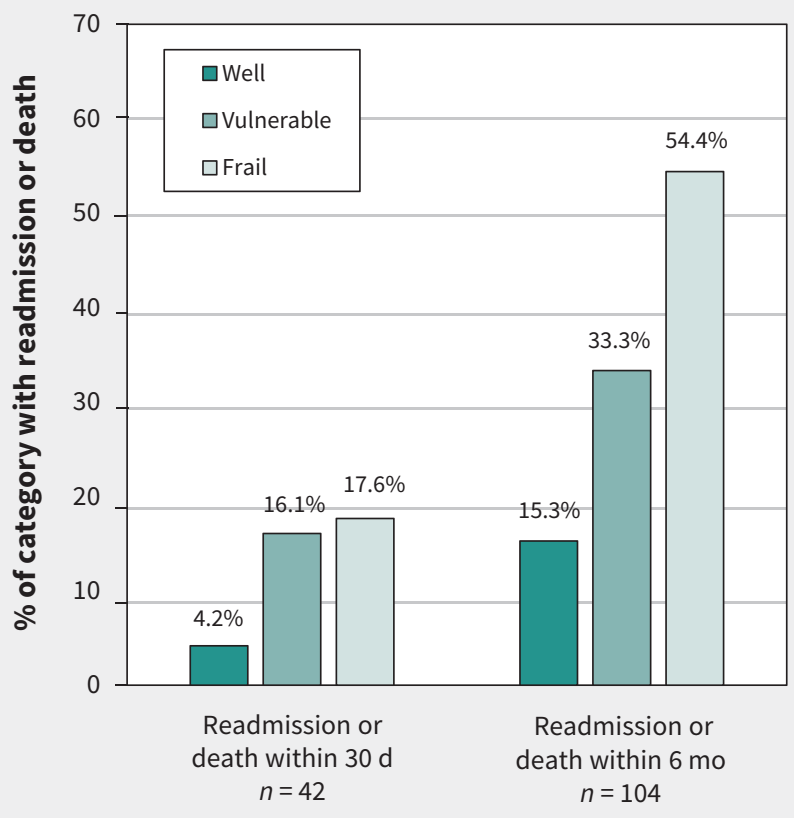

Figure 2: Relation between preadmission frailty and outcome after discharge. 
95\% Cl 1.01-4.55; for patients classified as frail, adjusted OR 3.27, $95 \% \mathrm{Cl} 1.32-8.12$; C statistic $0.75,95 \%$ Cl 0.69-0.81; Table 2).

\section{Sensitivity analyses}

A 1-point increase in the Clinical Frailty Scale predicted a greater risk of readmission or death, both independently (at $30 \mathrm{~d}$, adjusted OR $1.35,95 \% \mathrm{Cl} 1.03-1.77$, C statistic $0.74,95 \% \mathrm{Cl} 0.66-0.81$; at 6 mo, adjusted OR $1.39,95 \% \mathrm{Cl} 1.11-1.75$, C statistic $0.75,95 \% \mathrm{Cl}$ 0.69-0.81) and without adjustment (at $30 \mathrm{~d}$, crude $\mathrm{C}$ statistic 0.65 , 95\% Cl 0.56-0.73; at 6 mo, crude C statistic $0.68,95 \% \mathrm{Cl} 0.62-0.74$ ). Neither increasing age (at $30 \mathrm{~d}$, adjusted OR 0.76, 95\% Cl 0.47-1.23; at 6 mo, adjusted OR $0.98,95 \% \mathrm{Cl} 0.68-1.40$ ) nor a 1-point increase in the American Society of Anesthesiologists classification (at $30 \mathrm{~d}$, adjusted OR 1.17, 95\% Cl 0.69-1.98; at 6 mo, adjusted OR 1.00, 95\% $\mathrm{Cl}$ 0.67-1.49) was associated with readmission or death. A post hoc sensitivity analysis that excluded patients who underwent cancer surgery $(n=22,7.1 \%)$ did not change the positive association between frailty and readmission or death at 30 days or at 6 months (data not shown). Applying a Clinical Frailty Scale cut-off score of 4 yielded similar results to the tertile analyses (at $30 \mathrm{~d}$, adjusted OR 2.42, 95\% Cl 1.16-5.02; at 6 mo, adjusted OR 2.21, 95\% Cl 1.25-
3.92). Additional models were adjusted for the same confounders included in the final models above. With disaggregation of composite outcomes, we found that readmissions drove the observed associations (Table 2). Patients were most commonly readmitted for gastrointestinal problems, infection or pulmonary disease (at $30 \mathrm{~d}, 15 / 40$ [37.5\%], 8/40 [20.0\%] and 5/40 [12.5\%], respectively; at 6 mo, 32/99 [32.3\%], 19/99 [19.2\%] and 11/99 [11.1\%], respectively). No patients classified as well had died by 6 months after discharge.

\section{Interpretation}

This study had 3 main findings. First, one-third of older patients were readmitted or died within 6 months after discharge following surgery. Second, patients classified as vulnerable or frail were at increased risk of 30-day readmission or death. Third, by 6 months, the degree of frailty predicted, in a dose-dependent manner, increased risk of readmission or death after multivariable adjustment.

Our findings support and expand evidence concerning frailty and readmission. Of 25 studies on frailty assessment for prognosis

Table 2: Relation between preadmission frailty and outcome after discharge

\begin{tabular}{|c|c|c|c|}
\hline \multirow[b]{2}{*}{ Outcome* } & \multicolumn{3}{|c|}{ Preadmission level of frailty $\dagger$} \\
\hline & $\begin{array}{c}\text { Well } \\
n=72\end{array}$ & $\begin{array}{c}\text { Vulnerable } \\
n=168\end{array}$ & $\begin{array}{c}\text { Frail } \\
n=68\end{array}$ \\
\hline \multicolumn{4}{|c|}{ 30-day readmission or death $\ddagger$} \\
\hline No. $(\%)$ of patients & $3(4.2)$ & $27(16.1)$ & $12(17.6)$ \\
\hline Crude OR (95\% Cl) & 1.00 (ref) & $4.40(1.29-15.02)$ & $4.93(1.33-18.33)$ \\
\hline Adjusted OR (95\% Cl) & 1.00 (ref) & $4.60(1.29-16.45)$ & $4.51(1.13-17.94)$ \\
\hline \multicolumn{4}{|l|}{ 30-day readmission $\ddagger$} \\
\hline No. (\%) of patients & $3(4.2)$ & $26(15.5)$ & $11(16.2)$ \\
\hline Crude OR $(95 \% \mathrm{CI})$ & 1.00 (ref) & $4.21(1.23-14.49)$ & $4.44(1.18-16.68)$ \\
\hline Adjusted OR (95\% CI) & 1.00 (ref) & $4.23(1.20-14.97)$ & $3.97(1.00-15.78)$ \\
\hline \multicolumn{4}{|c|}{ 6-month readmission or death§ } \\
\hline No. (\%) of patients & $11(15.3)$ & $56(33.3)$ & $37(54.4)$ \\
\hline Crude OR (95\% Cl) & 1.00 (ref) & $2.77(1.35-5.68)$ & $6.62(2.97-14.73)$ \\
\hline Adjusted OR (95\% Cl) & 1.00 (ref) & $2.15(1.01-4.55)$ & $3.27(1.32-8.12)$ \\
\hline \multicolumn{4}{|l|}{ 6-month readmission§ } \\
\hline No. (\%) of patients & $11(15.3)$ & $55(32.7)$ & $33(48.5)$ \\
\hline Crude OR (95\% CI) & 1.00 (ref) & $2.70(1.32-5.54)$ & $5.23(2.35-11.63)$ \\
\hline Adjusted OR (95\% Cl) & 1.00 (ref) & $2.20(1.04-4.62)$ & $3.03(1.23-7.49)$ \\
\hline \multicolumn{4}{|c|}{$\begin{array}{l}\text { Note: } \mathrm{Cl}=\text { confidence interval, } \mathrm{OR}=\text { odds ratio. } \\
\text { *Age, sex and type of surgery were forced into all models. Additional variables meeting the statistical criteria for } \\
\text { confounding are detailed in separate footnotes for the individual models. } \\
\text { †Level of frailty based on Canadian Study of Health and Aging Clinical Frailty Scale. }{ }^{15} \\
\text { łFor the } 30 \text {-day model, the following variables met the statistical criteria for confounding: hemoglobin level on } \\
\text { admission, postoperative recovery on ward, postoperative use of total parenteral nutrition and Charlson Comorbidity } \\
\text { Index. The final } 30 \text {-day model was adjusted for age, sex, type of surgery, hemoglobin level on admission and } \\
\text { postoperative use of total parenteral nutrition. } \\
\text { \$For the 6-month model, the following variables met the statistical criteria for confounding: creatinine and hemoglobin } \\
\text { levels on admission, postoperative use of total parenteral nutrition, intraoperative ostomy creation and Charlson } \\
\text { Comorbidity Index. The final } 6 \text {-month model was adjusted for age, sex, type of surgery, hemoglobin level on admission, } \\
\text { intraoperative ostomy creation and the Charlson Comorbidity Index. }\end{array}$} \\
\hline
\end{tabular}


after cardiac surgery, only 2 used the Clinical Frailty Scale, and none used the scale to predict readmission. ${ }^{13}$ The handful of prior studies conducted in a surgical setting largely reported adverse prognosis for patients with frailty, but were mostly restricted to specific procedures and short follow-up periods. ${ }^{23-27}$ Very few studies have assessed a dose-response relation. Among 325 older patients who underwent general surgery, no difference was found in unadjusted 30-day readmission rates between frail and nonfrail patients, where frailty was defined by Clinical Frailty Scale scores of 5 or higher. ${ }^{23}$ However, in 383 adult kidney transplant recipients, frailty classified by the Fried criteria identified a $60 \%$ increased risk of 30 -day readmission. ${ }^{24}$ Furthermore, in a retrospective study of 5627 adults who underwent posterior cervical fusion, each additional point on a frailty-based index conferred a $40 \%$ increased risk of 30 -day readmission, ${ }^{25}$ similar to the $35 \%$ increase in risk per additional point in the Clinical Frailty Scale observed in our study. For 178 older patients with colorectal cancer, frailty on comprehensive geriatric assessment was associated with 2.5 times higher unadjusted risk of 30-day readmission related to colorectal surgery. ${ }^{26}$ Similarly, after classification of 72 older patients undergoing colorectal surgery on the basis of 7 frailty traits, 30-day readmission rates rose with increasing frailty from $6 \%$ to $29 \% .{ }^{27}$ Among nonsurgical patients, a Clinical Frailty Scale score of 5 or higher independently predicted a threefold risk of 30-day readmission or death among 245 older medical patients ${ }^{17}$ and a twofold risk of 1 -year readmission among 421 critically ill older patients. ${ }^{5}$

In this study, we overcame several limitations of prior studies by enrolling an older but largely unselected surgical cohort and reporting outcomes that occurred as long as 6 months after discharge. We also carefully assessed confounders, adjusting a priori for age, sex and type of surgery (because these factors have been linked to frailty and readmission) and examining a range of demographic, biological and clinical factors. Moreover, by describing the dose-response relation between degree of frailty and readmission rates, we provide evidence of risk in patients admitted without noticeable disability who had lower Clinical Frailty Scale scores. Although our cohort was relatively small compared with potential nonsurgical or all-aged groups, the patients were admitted to hospital for a wide range of abdominal diseases, which supports the importance of frailty in predicting readmission risk in various illnesses. Thus, the results may be relevant for clinicians or researchers concerned with prognosis in older patients in general.

Several mechanisms may explain the observed relation. Adverse outcomes could result from environmental or behavioural factors, particularly if extreme vulnerability to stressors like surgery ${ }^{28}$ increases the time of return to physiologic baseline, thereby predisposing frail patients to functional decline ${ }^{29}$ and reduced self-care capacity. ${ }^{30,31}$ Social factors may also modulate the adverse effects of frailty, whereby patients of lower socioeconomic status are both more likely to be classified as frail and more likely to be readmitted to hospital. ${ }^{32}$ Additionally, the inflammatory state associated with postoperative healing may exacerbate the already impaired immune system of frail older patients, ${ }^{33}$ thereby increasing postoperative infection rates ${ }^{34}$ or worsening comorbid conditions, leading to readmission. Alternatively, residual confounding may account for some of the risk observed; however, we adjusted for a variety of clinical and biologic markers, as did multiple prior studies of mortality. ${ }^{13,24,25}$

\section{Limitations}

Although this study featured two-centre prospective enrolment, extensive data collection and prolonged follow-up, it was limited by several factors. The findings cannot be generalized to severely frail or terminally ill patients because these groups were excluded. However, we enrolled a diverse cohort and we were more interested in less severely frail patients who are amenable to preventive strategies. Although we did not compare different tools or assess more objective frailty measures, we selected a frailty tool that is easy to use, reliable and predictive of clinically relevant outcomes, ${ }^{16-18}$ and that has been recommended for use in other surgical populations. ${ }^{13}$ We did not assess long-term disability or quality of life, which may be of particular importance to clinicians and patients. Lastly, because a dose-response relation was not observed at 30 days, it is possible that our 30-day results were limited by lower event rates or that frailty is more important for long-term than short-term prognosis.

\section{Conclusion}

Identifying frailty in surgical patients will help to predict which patients are at high risk of adverse outcomes, thus improving patient and family discussions and targeting patients for enhanced postoperative care. Moreover, the results of this study suggest that poor postoperative prognosis is not limited to the most severely frail patients, but that vulnerable patients without evident disability are also at higher risk of readmission or death after discharge. Further studies are needed to assess the impact and feasibility of interventions in terms of changing frailty status or decreasing risks among frail surgical patients, but current evidence supports the use of well-validated frailty assessments when evaluating risk for adverse postoperative outcomes.

\section{References}

1. All-cause readmission to acute care and return to the emergency department. Ottawa: Canadian Institute for Health Information; 2012.

2. Readmissions reduction program (HRRP). Baltimore: Centers for Medicare \& Medicaid Services; 2016. Available: https://www.cms.gov/Medicare/Medicare-Fee -for-Service-Payment/AcutelnpatientPPS/Readmissions-Reduction-Program.html (accessed 2016 Nov. 8).

3. Population projections for Canada, provinces and territories: 2009 to 2036. Cat no 91-520-X. Ottawa: Statistics Canada; 2010. Available: www.statcan.gc.ca/ pub/91-520-x/91-520-x2010001-eng.pdf (accessed 2016 Nov. 8).

4. Holt PJE, Poloniecki JD, Hofman D, et al. Re-interventions, readmissions and discharge destination: modern metrics for the assessment of the quality of care. Eur J Vasc Endovasc Surg 2010;39:49-54.

5. Bagshaw SM, Stelfox HT, McDermid RC, et al. Association between frailty and short- and long-term outcomes among critically ill patients: a multicentre prospective cohort study. CMAJ 2014;186:E95-102.

6. Rockwood K, Bergman H. FRAILTY: a report from the 3rd joint workshop of IAGG/WHO/SFGG, Athens, January 2012. Can Geriatr J 2012;15:31-6.

7. Partridge JSL, Harari D, Dhesi JK. Frailty in the older surgical patient: a review. Age Ageing 2012;41:142-7.

8. Makary MA, Segev DL, Pronovost PJ, et al. Frailty as a predictor of surgical outcomes in older patients. J Am Coll Surg 2010;210:901-8. 
9. Dasgupta M, Rolfson DB, Stolee P, et al. Frailty is associated with postoperative complications in older adults with medical problems. Arch Gerontol Geriatr 2009;48:78-83.

10. Farhat JS, Velanovich V, Falvo AJ, et al. Are the frail destined to fail? Frailty index as predictor of surgical morbidity and mortality in the elderly. J Trauma Acute Care Surg 2012;72:1526-30, discussion 1530-1.

11. Joseph B, Pandit V, Zangbar B, et al. Superiority of frailty over age in predicting outcomes among geriatric trauma patients: a prospective analysis. JAMA Surg 2014;149:766-72.

12. Lee DH, Buth KJ, Martin BJ, et al. Frail patients are at increased risk for mortality and prolonged institutional care after cardiac surgery. Circulation 2010; 121:973-8.

13. Kim DH, Kim CA, Placide S, et al. Preoperative frailty assessment and outcomes at 6 months or later in older adults undergoing cardiac surgical procedures: a systematic review. Ann Intern Med 2016;165:650-60.

14. Revenig LM, Canter DJ, Henderson MA, et al. Preoperative quantification of perceptions of surgical frailty. J Surg Res 2015;193:583-9.

15. Clinical frailty scale. Version 1.2. Halifax: Dalhousie University, Geriatric Medicine Research; 2007-2009. Available: http://geriatricresearch.medicine.dal.ca/ pdf/Clinical\%20Faily\%20Scale.pdf (accessed 2017 Mar. 6).

16. Rockwood K, Song X, Macknight $\mathrm{C}$, et al. A global clinical measure of fitness and frailty in elderly people. CMAJ 2005;173:489-95.

17. Belga S, Majumdar SR, Kahlon S, et al. Comparing three different measures of frailty in medical inpatients: multicenter prospective cohort study examining 30-day risk of readmission or death. J Hosp Med 2016;11:556-62.

18. Ritt M, Bollheimer LC, Sieber CC, et al. Prediction of one-year mortality by five different frailty instruments: a comparative study in hospitalized geriatric patients. Arch Gerontol Geriatr 2016;66:66-72.

19. Khadaroo RG, Padwal RS, Wagg AS, et al. Optimizing senior's surgical care Elder-friendly Approaches to the Surgical Environment (EASE) study: rationale and objectives. BMC Health Serv Res 2015;15:338.

20. Frenkel WJ, Jongerius EJ, Mandjes-van Uitert MJ, et al. Validation of the Charlson Comorbidity Index in acutely hospitalized elderly adults: a prospective cohort study. J Am Geriatr Soc 2014;62:342-6.
21. Babyak MA. What you see may not be what you get: a brief, nontechnical introduction to overfitting in regression-type models. Psychosom Med 2004;66:411-21.

22. Kahlon S, Pederson J, Majumdar SR, et al. Association between frailty and 30-day outcomes after discharge from hospital. CMAJ 2015;187:799-804.

23. Hewitt J, Moug SJ, Middleton M, et al.; Older Persons Surgical Outcomes Collaboration. Prevalence of frailty and its association with mortality in general surgery. Am J Surg 2015;209:254-9.

24. McAdams-DeMarco MA, Law A, Salter ML, et al. Frailty and early hospital readmission after kidney transplantation. Am J Transplant 2013;13:2091-5.

25. Medvedev G, Wang C, Cyriac M, et al. Complications, readmissions, and reoperations in posterior cervical fusion. Spine 2016;41:1477-83.

26. Kristjansson SR, Nesbakken A, Jordhøy MS, et al. Comprehensive geriatric assessment can predict complications in elderly patients after elective surgery for colorectal cancer: a prospective observational cohort study. Crit Rev Oncol Hematol 2010;76:208-17.

27. Robinson TN, Wu DS, Pointer L, et al. Simple frailty score predicts postoperative complications across surgical specialties. Am J Surg 2013;206:544-50.

28. Fried LP, Hadley EC, Walston JD, et al. From bedside to bench: research agenda for frailty. Sci Aging Knowledge Environ 2005;2005:pe24.

29. Gregorevic KJ, Hubbard RE, Lim WK, et al. The clinical frailty scale predicts functional decline and mortality when used by junior medical staff: a prospective cohort study. BMC Geriatr 2016;16:117.

30. Uchmanowicz I, Wleklik M, Gobbens RJJ. Frailty syndrome and self-care ability in elderly patients with heart failure. Clin Interv Aging 2015;10:871-7.

31. Gazala S, Tul Y, Wagg A, et al. Quality of life and long-term outcomes of octoand nonagenarians following acute care surgery: a cross sectional study. World J Emerg Surg 2013;8:23.

32. Gutiérrez-Robledo LM, Avila-Funes JA. How to include the social factor for determining frailty? J Frailty Aging 2012;1:13-7.

33. Wang GC, Casolaro V. Immunologic changes in frail older adults. Transl Med UniSa 2014;9:1-6.

34. Berlin A, Johanning JM. Intraabdominal infections in older adults. Clin Geriatr Med 2016;32:493-507.

\section{Competing interests: None declared.}

This article has been peer reviewed.

Affiliations: Departments of Surgery (Li, Pederson, Churchill, Khadaroo), Medicine (Wagg, Alagiakrishnan, Padwal) and Critical Care Medicine (Khadaroo), University of Alberta; Alberta Seniors Health Strategic Clinical Network (Wagg, Alagiakrishnan), Alberta; Departments of Medicine (Holroyd-Leduc) and Community Health Sciences (HolroydLeduc), University of Calgary, Calgary, Alta.; Alberta Diabetes Institute (Padwal), Edmonton, Alta.

Contributors: Yibo Li and Jenelle Pederson contributed equally as first authors. All of the authors contributed to the design and conduct of the study. Yibo Li and Jenelle Pederson were responsible for data collection and analysis, and drafted the first version of the manuscript. Rachel Khadaroo supervised the study. All of the authors critically reviewed the manuscript, gave final approval of the version to be published and agreed to be accountable for all aspects of the work.
Funding: The Elder-friendly Approaches to the Surgical Environment (EASE) study is funded by the Alberta Innovates Health Solutions Partnership for Research and Innovation in the Health System grant 201300465.

Data sharing: This substudy is based on pre-implementation data for the Elder-friendly Approaches to the Surgical Environment (EASE) study, which is not yet complete. Data can be shared when the main study has been finished and its results published.

Acknowledgements: The authors would like to thank Drs. Fiona Clement, Heather Hanson and Susan Slaughter for continued support of their research and Lindsey M. Warkentin, Ashley Wanamaker, Carrie Le and Hanhmi Huynh for their contributions to data collection for this study.

Disclaimer: Jayna Holroyd-Leduc is an associate editor for CMAJ and was not involved in the editorial decision-making process for this article.

Accepted: July 11, 2017

Correspondence to: Rachel Khadaroo, khadaroo@ualberta.ca 